\title{
The Impact of COVID-19 Immunity in Vaccine Development
}

\author{
Masoud Mardani (ii) ${ }^{1, *}$ \\ ${ }^{1}$ Infectious Diseases and Tropical Medicine Research Center, Shahid Beheshti University of Medical Sciences, Tehran, Iran \\ "Corresponding author: Infectious Diseases and Tropical Medicine Research Center, Shahid Beheshti University of Medical Sciences, Tehran, Iran. Tel: +98-9121132678, Email: \\ drmasoudmardani@yahoo.com
}

Received 2020 September 29; Accepted 2020 September 29.

Keywords: Vaccine, COVID-19, Immunity

The process of development of immunity to pathogen through natural infection has multi-steps, which typically takes 1 - 2 weeks. As the body's first line of defense, the immune system responds to foreign substances. In which macrophages, neutrophils, and dendritic cells come to work to either slow down the progress of the virus or, in some cases, to suppress it. The innate immune system has the ability to "remember" foreign substances, so the next step is producing antibodies (proteins that are also known as immunoglobulin) to make the response faster. In addition, the body also produces T-cells that detect and destroy cells infected with viruses, which is called cellular immunity. Therefore, triggering the adaptive immunity by an infection stimulates the body's response that results in either elimination of the virus or preventing severe illnesses, while protecting the body against reinfection. As the presence of antibody indicates previous infection, it's a criterion to measure the immunological response (1).

Severe acute respiratory syndrome coronavirus 2 (SARS-CoV-2) stimulates the adaptive immunity response within the first 7 - 10 days of infection. To forecast the prognosis of the disease and develop effective strategies to control the pandemic, first, we should understand the key features and evolution of B-cell and T-cell mediated immunity to SARS-CoV-2. Moreover, it is of crucial importance to understand the durable protection, which may guaranty long-term B-cell and T-cell immunological memory against SARS-CoV-2 (2-4).

Five to seven days after onset of infection, the immune system stimulates to produce serum IgM and IgA, and in 7 10 days, IgG level begins to rise, which altogether cause a robust memory B-cell. Therefore, plasmablast expansion is detectable in early infection. Then, in nearly 28 days, serum IgM and IgA titers begin to decline, and IgG titers peak at approximately 49 days after infection. In the first week of infection with SARS-CoV-2 concurrently activates Tcells and virus-specific memory $\mathrm{CD} 4^{+}$cells and $\mathrm{CD} 8^{+}$seemingly peak within 2 weeks but remains detectable for 100 or more days of observation at lower levels. Based on the currently available data, SARS-CoV-2 specific memory $\mathrm{CD} 4^{+}$ T-cells is detectable in all patients recovered from the disease, while $\mathrm{CD} 8{ }^{+} \mathrm{T}$-cells are detectable in about $70 \%$ of them (5). Also, it seems that antibody-dependent enhancement or dominant $\mathrm{CD}^{+}{ }^{+}$TH2-type cytokine (IL-4, IL-5, IL-13) do not contribute to the severity of infection with COVID-19, despite the existence of high viral titers, a dysregulated innate inflammatory cytokine, chemokine response, and prolonged lymphopenia. The intensity of the antibody and T-cells vary from person to person and is dependent on the severity of the diseases (that is, asymptomatic, mild, moderate, severe).

Correlates of protection for COVID-19 are not established yet, but neutralizing antibodies, especially those that recognize viral receptor-binding domain (RBD) and other epitopes on the spike protein (which prevent succeeding angiotensin-converting enzyme II receptor binding, membrane fusion, and viral entry), is one path to immunity (5-7). To control the COVID-9 pandemic and neutralize the virus, first, we should understand antibody responses which developed following the infection, mainly through building immune response to the spike protein that aid in blocking the entry of the virus to the cell. In animal SARS-CoV-2 infection models, such virus neutralizing antibodies have yielded protective results.

It's believed that protection against SARS-CoV-2 in nonhuman primates is mediated by neutralizing antibodies induced by $\mathrm{TH} 1$ biased $\mathrm{CD}^{+}{ }^{+} \mathrm{T}$-cell response. Although SARS-COV-2 is an emerging virus that we know little about 
it, it has been proved that there is little sequence homology of SARS-CoV-2 spike protein to the seasonal coronaviruses (3-5).

About one-third of patients recovered from COVID-19 didn't have antibodies with low anti-RBD titers and low viral neutralizing activity, especially those with mild or asymptomatic diseases. Various factors contribute to variability in antibody responses. Patients with a severe disease often have the highest neutralizing antibody titers, a major driver of the magnitude of the response is antigen burden $(4,5)$.

We are eight months into the pandemic, and it has claimed millions of lives, and various studies are published about infection and reinfection with COVID-19. Although the immunity profile of recovered patients isn't clear, high rates of reinfection indicate that the common human immune response may not give sterilizing immunity. However, it might reduce viral shedding, decrease spread, and prevent infection (5). Considerable information now demonstrates the preexisting T-cell immunity to SARS-CoV-2 in blood donors either before the coronavirus pandemic or recently among those without the infection. Memory $\mathrm{CD}^{+} \mathrm{T}$ cells are found in higher frequencies than $\mathrm{CD}^{+} \mathrm{T}$ cells, and this feasible speak to reactions actuated by the past disease with other human endemic beta coronaviruses known to cause the common cold $(2,5,6)$.

Based on the Seroprevalence information, the true number of infected people with SARS-CoV-2 worldwide may be 10 times higher than reported cases (probably 40 to 50 million people are infected). Although depending on populace based natural immunity, particularly for highrisk groups, it is not astute. However, measures such as boosting specific neutralizing antibodies and TH1 immunity and paying attention to earlier immunity status may additionally protect these people (5).

Coronavirus immunizations being developed intended to forestall clinical contamination, disease severity, or both show the enlistment of an anamnestic insusceptible response to the spike protein with a subsequent dose and can produce elevated levels of neutralizing antibodies equivalent to or more prominent than those seen in sera sample from patients $(5,7)$.

Although COVID-19 is now a pandemic, but it may follow the way of previous coronaviruses and get endemic. Hence, in the few "short" months since the appearance of the virus, 2 key paths to COVID-19 adaptive immunity are being unraveled, therefore we should use these information to create immunization (5).

To sum up, by employing the available experiences and data on immunity to COVID-19 as well as protective immunity to this virus, we can generate a comprehensive solution to develop an efficient and effective vaccine in the future.

\section{Footnotes}

Conflict of Interests: The author declares no conflict of interest.

Funding/Support: None declared by author.

\section{References}

1. WHO. 2020. Available from: www.who.int.

2. Grifoni A, Weiskopf D, Ramirez SI, Mateus J, Dan JM, Moderbacher $\mathrm{CR}$, et al. Targets of $\mathrm{T}$ Cell Responses to SARS-CoV-2 Coronavirus in Humans with COVID-19 Disease and Unexposed Individuals. Cell. 2020;181(7):1489-1501 e15. doi: 10.1016/j.cell.2020.05.015. [PubMed: 32473127]. [PubMed Central: PMC7237901].

3. Suthar MS, Zimmerman MG, Kauffman RC, Mantus G, Linderman SL, Hudson WH, et al. Rapid Generation of Neutralizing Antibody Responses in COVID-19 Patients. Cell Rep Med. 2020;1(3):100040. doi 10.1016/j.xcrm.2020.100040. [PubMed: 32835303]. [PubMed Central: PMC7276302].

4. Robbiani DF, Gaebler C, Muecksch F, Lorenzi JCC, Wang Z, Cho A, et al. Convergent antibody responses to SARS-CoV-2 in convalescent individuals. Nature. 2020;584(7821):437-42. doi: 10.1038/s41586-020-24569. [PubMed: 32555388]. [PubMed Central: PMC7442695].

5. Stephens DS, McElrath MJ. COVID-19 and the Path to Immunity. JAMA 2020. doi: 10.1001/jama.2020.16656. [PubMed: 32915201].

6. Le Bert N, Tan AT, Kunasegaran K, Tham CYL, Hafezi M, Chia A, et al. SARS-CoV-2-specific $T$ cell immunity in cases of COVID-19 and SARS, and uninfected controls. Nature. 2020;584(7821):457-62. doi: 10.1038/s41586-020-2550-z. [PubMed: 32668444].

7. Braun J, Loyal L, Frentsch M, Wendisch D, Georg P, Kurth F, et al. SARSCoV-2-reactive T cells in healthy donors and patients with COVID-19. Nature. 2020. doi: 10.1038/s41586-020-2598-9. 